Homology, Homotopy and Applications, vol.16(2), 2014, pp.45-64

\title{
DERIVED CATEGORIES OF ABSOLUTELY FLAT RINGS
}

\author{
GREG STEVENSON
}

\author{
(communicated by J. Daniel Christensen)
}

\begin{abstract}
Let $S$ be a commutative ring with topologically noetherian spectrum, and let $R$ be the absolutely flat approximation of $S$. We prove that subsets of the spectrum of $R$ parametrise the localising subcategories of $\mathrm{D}(R)$. Moreover, we prove the telescope conjecture holds for $\mathrm{D}(R)$. We also consider unbounded derived categories of absolutely flat rings that are not semiartinian and exhibit a localising subcategory that is not a Bousfield class and a cohomological Bousfield class that is not a Bousfield class.
\end{abstract}

\section{Introduction}

Given a commutative noetherian ring $S$, the structure of the unbounded derived category $\mathrm{D}(S)$ and its full subcategory of compact objects $\mathrm{D}^{\text {perf }}(S)$ is very well understood. By a result of Hopkins and Neeman [Nee92] the lattice of thick subcategories of $\mathrm{D}^{\text {perf }}(S)$ is isomorphic to the lattice of specialisation closed subsets of Spec $S$. Neeman proves in [Nee92, Theorem 2.8] that when one passes to $\mathrm{D}(S)$ this extends to a lattice isomorphism between arbitrary subsets of $\operatorname{Spec} S$ and localising subcategories of $\mathrm{D}(S)$.

Now suppose $S$ is commutative but not noetherian. Then, by work of Thomason [Tho97, Theorem 3.15], the classification of thick subcategories of $\mathrm{D}^{\mathrm{perf}}(S)$ is still valid; there is a lattice isomorphism between thick subcategories of $\mathrm{D}^{\text {perf }}(S)$ and Thomason subsets of Spec $S$ i.e., those subsets that can be written as unions of closed subsets with quasi-compact complements. However, we are almost completely ignorant concerning the structure of $\mathrm{D}(S)$; we do not even know if the collection of localising subcategories forms a set rather than a proper class. Some partial results, indicating the possible complexity of the lattice of localising subcategories, have been obtained by Neeman [Nee00] and then in later work of Dwyer and Palmieri [DP08] for graded non-noetherian truncated polynomial rings.

In this work we consider the situation for a commutative absolutely flat ring $R$ i.e., $R$ is commutative, reduced, and zero dimensional. Such rings are also called von Neumann regular rings, but, we will favour the "geometric" name and not use this

This research was partly supported by a fellowship from the Alexander von Humboldt Foundation. Received August 5, 2013, revised April 14, 2014; published on July 21, 2014.

2010 Mathematics Subject Classification: 18E30, 16E50.

Key words and phrases: derived category, absolutely flat ring, localising subcategory, telescope conjecture.

Article available at http://dx.doi.org/10.4310/HHA.2014.v16.n2.a3

Copyright (C) 2014, International Press. Permission to copy for private use granted. 
terminology. We observe two starkly contrasting ways in which the derived category of such a ring can behave. If $R$ is not semi-artinian, we show in Theorem 4.8 that the residue fields of $R$ do not generate the derived category, and as a corollary we exhibit a localising subcategory that cannot be realised as the kernel of tensoring by any object of $\mathrm{D}(R)$ (Corollary 4.10). This gives a counterexample to the analogue of a conjecture of Hovey and Palmieri [HP99, Conjecture 9.1] (concerning the stable homotopy category) in the setting of derived categories of rings. In a similar vein we give in Corollary 4.12 an example of a cohomological Bousfield class that is not a Bousfield class (this terminology is explained in Definition 4.9).

On the other hand, suppose $S$ is a commutative ring with topologically noetherian spectrum. The absolutely flat approximation of $S$ is an absolutely flat ring $S^{\text {abs }}$ together with a morphism $S \longrightarrow S^{\text {abs }}$ through which all other morphisms from $S$ to an absolutely flat ring factor. We prove in Theorem 4.23 that Neeman's classification is valid for $\mathrm{D}\left(S^{\text {abs }}\right)$ : there is a set of localising subcategories, and the lattice they form is isomorphic to the powerset of Spec $S^{\text {abs }}$. Furthermore, every localising subcategory of $\mathrm{D}\left(S^{\text {abs }}\right)$ is the kernel of tensoring with a module (Corollary 4.24) and the telescope conjecture holds (see Definition 4.17 and Theorem 4.25).

Now let us very briefly sketch the contents of the paper. Section 2 contains abstract results on tensor triangular geometry and support theory. More specifically, after some brief recollections on supports, we prove some results concerning the behaviour of supports under exact monoidal functors (Section 2.2) and give a topological restriction on the supports of quotients by smashing subcategories (Section 2.3); if the reader is mainly interested in the results concerning derived categories, she can safely skip or refer back to this section. Section 3 contains some preliminary material on absolutely flat rings and absolutely flat approximations. The main results concerning derived categories are proved in Section 4.

\section{Acknowledgments}

I am grateful to Jesse Burke and Mike Prest for helpful comments relating to this work. Many thanks are also due to Ivo Dell'Ambrogio for several helpful comments on a preliminary version of this article.

\section{Some tensor triangular abstract nonsense}

\subsection{Preliminaries}

We begin by briefly discussing some notions and notation that we will use throughout. We assume some familiarity with the subject matter; for further details and definitions the interested reader should consult $[\mathbf{B a l 0 5}]$ and $[\mathbf{B F 1 1}]$. Let $(\mathcal{K}, \otimes, \mathbf{1})$ be an essentially small rigid tensor triangulated category; i.e., $\mathcal{K}$ is a triangulated category with a compatible closed symmetric monoidal structure and for every $k, l \in \mathcal{K}$ the natural map

$$
\operatorname{hom}(k, \mathbf{1}) \otimes l \longrightarrow \operatorname{hom}(k, l)
$$

is an isomorphism, where $\mathbf{1}$ is the unit object and hom $(-,-)$ is the internal hom. 
Following [Bal05], we associate to $\mathcal{K}$ its spectrum $\mathrm{Spc} \mathcal{K}$. We recall that

$$
\operatorname{Spc} \mathcal{K}=\{\mathcal{P} \subsetneq \mathcal{K} \mid \mathcal{P} \text { is prime }\} .
$$

Here $\mathcal{P}$ is prime if $\mathcal{P}$ is a proper thick tensor ideal of $\mathcal{K}$ such that whenever $k \otimes l \in \mathcal{P}$, for $k, l \in \mathcal{K}$, we have $k \in \mathcal{P}$ or $l \in \mathcal{P}$. The Zariski topology on Spc $\mathcal{K}$ is given by the basis of closed subsets

$$
\{\operatorname{supp} k=\{\mathcal{P} \in \operatorname{Spc} \mathcal{K} \mid k \notin \mathcal{P}\} \mid k \in \mathcal{K}\} .
$$

This topology makes $\mathrm{Spc} \mathcal{K}$ into a spectral space; i.e., $\mathrm{Spc} \mathcal{K}$ is quasi-compact, $T_{0}$, every irreducible closed subset of $\mathrm{Spc} \mathcal{K}$ has a (necessarily unique) generic point, and the collection of quasi-compact open subsets of Spc $\mathcal{K}$ is closed under finite intersections and gives a basis of open subsets for the Zariski topology. Before proceeding, let us recall a few notions and facts about spectral spaces that will be needed in the sequel. Suppose $X$ and $Y$ are spectral spaces. A spectral map, or morphism of spectral spaces, is a continuous map $f: X \longrightarrow Y$ such that the preimage of any open quasi-compact subset of $Y$ under $f$ is again quasi-compact. We say a subset $\mathcal{V}$ of $X$ is Thomason if $\mathcal{V}$ can be written as a union of closed subsets of $X$ each of which has quasi-compact complement. It is immediate that given a spectral map $f: X \longrightarrow Y$ the preimage of a Thomason subset of $Y$ is again Thomason. We will also need to consider the following refinement of the topology on a spectral space.

Definition 2.1. Let $X$ be a spectral space. We denote by $X^{\text {con }}$ the set $X$ equipped with the constructible topology, which is given by taking the quasi-compact open subsets of $X$ and their complements as a subbasis of open sets.

A subset $Z$ of $X$ that is closed in the constructible topology is called proconstructible.

The topology on $X^{\text {con }}$ is also known as the patch topology; for instance, this is the terminology used in [Hoc69]. It is again a spectral space and is Hausdorff. We can characterise those subspaces of a spectral space $X$ that are again spectral with spectral inclusion in terms of the constructible topology. Indeed, a subset $Z$ of $X$ is proconstructible if and only if $Z$, endowed with the subspace topology, is spectral and the inclusion $Z \longrightarrow X$ is a spectral map. Further details and references concerning spectral spaces can be found, for instance, in [Tre06, Section 2].

Given another essentially small rigid tensor triangulated category $\mathcal{K}^{\prime}$ and an exact monoidal functor $F: \mathcal{K} \longrightarrow \mathcal{K}^{\prime}$, there is an induced continuous map

$$
\operatorname{Spc} F: \operatorname{Spc} \mathcal{K}^{\prime} \longrightarrow \operatorname{Spc} \mathcal{K}
$$

given by sending $\mathcal{P} \in \operatorname{Spc} \mathcal{K}^{\prime}$ to its preimage $F^{-1} \mathcal{P}$ under $F$. The map $\operatorname{Spc} F$ is spectral.

We also recall there is, by [Bal05, Theorem 4.10], a bijection between Thomason subsets of Spc $\mathcal{K}$ and thick tensor ideals of $\mathcal{K}$ sending a Thomason subset $\mathcal{V}$ to the ideal $\mathcal{K}_{\mathcal{V}}$ of all objects whose support is contained in $\mathcal{V}$.

Now suppose $(\mathcal{S}, \otimes, \mathbf{1})$ is a rigidly-compactly generated tensor triangulated category; i.e., $\mathcal{S}$ is a compactly generated triangulated category with a compatible closed symmetric monoidal structure such that the compact objects $\mathcal{S}^{c}$ form a rigid tensor triangulated subcategory. We denote, for $\mathcal{V} \subseteq \operatorname{Spc} \mathcal{S}^{c}$ a Thomason subset, by $\Gamma_{\mathcal{V}} \mathcal{S}$ the localising ideal generated by $\mathcal{S}_{\mathcal{V}}^{c}$, the thick ideal of compact objects supported on $\mathcal{V}$. 
The subcategory $\Gamma_{\mathcal{V}} \mathcal{S}$ is a smashing subcategory of $\mathcal{S}$, and the corresponding Rickard idempotents, giving rise to the acyclisation and localisation functors with respect to $\Gamma_{\mathcal{V}} \mathcal{S}$, will be denoted by $\Gamma_{\mathcal{V}} \mathbf{1}$ and $L_{\mathcal{V}} \mathbf{1}$, respectively.

Given $x \in \operatorname{Spc} \mathcal{S}^{c}$, we, as usual, set $\mathcal{V}(x)=\overline{\{x\}}$ and

$$
\mathcal{Z}(x)=\{y \mid x \notin \mathcal{V}(y)\} .
$$

A point $x$ of $\operatorname{Spc} \mathcal{S}^{c}$ is said to be visible if there exist Thomason subsets $\mathcal{V}, \mathcal{W}$ of Spc $\mathcal{S}^{c}$ such that $\mathcal{V} \backslash(\mathcal{V} \cap \mathcal{W})=\{x\}$. This is not the same definition as given in [BF11]; cf. Remark 4.3 for further details. In this case we set

$$
\Gamma_{x} \mathbf{1}=\Gamma_{\mathcal{V}} \mathbf{1} \otimes L_{\mathcal{W}} \mathbf{1}
$$

and recall from [BF11, Corollary 7.5] that up to isomorphism $\Gamma_{x} \mathbf{1}$ does not depend on the choice of $\mathcal{V}$ and $\mathcal{W}$.

Remark 2.2. With notation as above we can always choose $\mathcal{W}=\mathcal{Z}(x)$. Indeed, $\mathcal{Z}(x)$ is always Thomason (by virtue of being the support of the prime ideal of $\mathcal{S}^{c}$ corresponding to the point $x$ ), and $x \notin \mathcal{W}$ combined with the fact that $\mathcal{W}$ is specialisation closed implies $\mathcal{W} \subseteq \mathcal{Z}(x)$.

Denoting by $\operatorname{Vis} \mathcal{S}^{c}$ the set of visible points in $\operatorname{Spc} \mathcal{S}^{c}$, we define the big support of $X \in \mathcal{S}$ to be

$$
\operatorname{Supp} X=\left\{x \in \operatorname{Vis} \mathcal{S}^{c} \mid \Gamma_{x} \mathbf{1} \otimes X \neq 0\right\} .
$$

The big support is a subset of Vis $\mathcal{S}^{c}$, which can be a proper subset of $\operatorname{Spc} \mathcal{S}^{c}$. Thus we cannot in general have, for $s \in \mathcal{S}^{c}$, an equality between $\operatorname{supp} s$ and $\operatorname{Supp} s$. However, no information is lost in the sense that the big support of a compact object determines the usual support.

Lemma 2.3. Let $s$ be a compact object of $\mathcal{S}$. Then there is an equality

$$
\operatorname{Supp} s=\operatorname{supp} s \cap \operatorname{Vis} \mathcal{S}^{c} .
$$

Proof. Using Remark 2.2, one can mimic the proof of [BF11, Proposition 7.17].

Proposition 2.4. Let $s$ be a compact object of $\mathcal{S}$. Then there is an equality

$$
\overline{\operatorname{Supp} s}=\operatorname{supp} s .
$$

Proof. Let $x$ be a point of $\operatorname{supp} s \backslash \mathrm{Vis} \mathcal{S}^{c}$. By the lemma it is sufficient to show there is a visible point of $\operatorname{supp} s$ specialising to $x$. As $\operatorname{supp} s$ is a closed subspace of $\operatorname{Spc} \mathcal{S}^{c}$, it is a spectral space in its own right. Thus $x$ is a specialisation of a point $y \in \operatorname{supp} s$ that is minimal with respect to specialisation in $\operatorname{supp} s$. To complete the argument it is sufficient to show that $y$ is visible. This is the case since supp $s$ is Thomason and $\operatorname{supp} s \backslash \mathcal{Z}(y)=\{y\}$ by minimality of $y$ with respect to specialisation in $\operatorname{supp} s$.

Thus there is no real danger of confusing supp and Supp at the level of compact objects, so we will generally refer to the big support simply as the support and write supp for both. Of course, the reader may just instead assume that all points of the spectra we consider are visible - this will be the case in our applications. 


\subsection{Pullbacks of Rickard idempotents}

We now prove some facts concerning the behaviour of supports for rigidly-compactly generated tensor triangulated categories with respect to exact monoidal functors. The results are essentially what one would expect in analogy with the case of compact objects. The material in this section is used in Section 2.3 and, more concretely, in Section 4.2 to identify the images of certain Rickard idempotents under derived base change along maps of rings.

We are interested in the following setup: $\mathcal{S}$ and $\mathcal{T}$ are rigidly-compactly generated tensor triangulated categories and $F: \mathcal{S} \longrightarrow \mathcal{T}$ is a coproduct and compact object preserving monoidal functor. We denote the right adjoint of $F$, which exists by Brown representability, by $G$. We note that $G$ necessarily preserves coproducts; see, for instance, [Nee96, Theorem 5.1]. We want to consider what $F$ does to the Rickard idempotents of [BF11]; our setup is modelled on the situation of pulling back along a quasi-compact quasi-separated map of schemes. Our results extend those in Section 8 of $[\mathbf{S t e 1 3}]$ to situations more general than localisations.

Notation 2.5. Given a class of objects $M$ in $\mathcal{S}$, we will denote by $\langle M\rangle$ and $\langle M\rangle_{\otimes}$ the smallest localising subcategory and the smallest localising ideal containing $M$, respectively.

In situations where we wish to distinguish via notation, rather than context, in which category a tensor product is being taken we will use subscripts - e.g., $\otimes_{\mathcal{S}}$ or $\otimes_{\mathcal{T}}$ - to be completely clear, and similarly for the support and tensor units.

Let us denote by $F^{c}$ the restriction of $F$ to compacts, i.e.,

$$
F^{c}: \mathcal{S}^{c} \longrightarrow \mathcal{T}^{c},
$$

which exists by assumption. We obtain a spectral (i.e., quasi-compact) map of spectral spaces

$$
\operatorname{Spc}\left(F^{c}\right)=f: \operatorname{Spc} \mathcal{T}^{c} \longrightarrow \operatorname{Spc} \mathcal{S}^{c},
$$

and we know, by [Bal05, Proposition 3.6], that $\operatorname{supp} F s=f^{-1} \operatorname{supp} s$ for all $s \in \mathcal{S}^{c}$. In particular, $F s=0$ if and only if $f^{-1} \operatorname{supp} s=\varnothing$.

As $f: \operatorname{Spc} \mathcal{T}^{c} \longrightarrow \operatorname{Spc} \mathcal{S}^{c}$ is spectral, $f^{-1}$ sends Thomason subsets to Thomason subsets. This leads to the following observation.

Lemma 2.6. Let $\mathcal{V}$ be a Thomason subset of $\mathrm{Spc} \mathcal{S}^{c}$. There are equalities of localising ideals of $\mathcal{T}$

$$
\left\langle F \Gamma_{\mathcal{V}} \mathcal{S}\right\rangle_{\otimes}=\left\langle F \mathcal{S}_{\mathcal{V}}^{c}\right\rangle_{\otimes}=\Gamma_{f^{-1}} \mathcal{V} \mathcal{T}
$$

Proof. The first equality is easily checked-for instance, it follows from [Ste13, Lemma 3.8]. We prove the second equality. It is clear from the formula

$$
\operatorname{supp} F s=f^{-1} \operatorname{supp} s
$$

for $s \in \mathcal{S}^{c}$ that $F \mathcal{S}_{\mathcal{V}}^{c} \subseteq \mathcal{T}_{f^{-1} \mathcal{V}}^{c}$ and so $\left\langle F \mathcal{S}_{\mathcal{V}}^{c}\right\rangle_{\otimes} \subseteq \Gamma_{f^{-1}} \mathcal{V} \mathcal{T}$. In fact, one even sees from the support formula that $\operatorname{supp} F \mathcal{S}_{\mathcal{V}}^{c}=f^{-1} \mathcal{V}$. By Balmer's classification result [Bal05, Theorem 4.10] we thus deduce that the smallest localising tensor ideal containing $F \mathcal{S}_{\mathcal{V}}^{c}$ contains $\mathcal{T}_{f^{-1} \mathcal{V}}^{c}$ and hence contains $\Gamma_{f^{-1}} \mathcal{V} \mathcal{T}$. 
Proposition 2.7. Let $\mathcal{V}$ be a Thomason subset of $\operatorname{Spc} \mathcal{S}^{c}$. Then there are natural isomorphisms of Rickard idempotents

$$
F\left(\Gamma_{\mathcal{V}} \mathbf{1}_{\mathcal{S}}\right) \cong \Gamma_{f^{-1} \mathcal{V}} \mathbf{1}_{\mathcal{T}} \quad \text { and } \quad F\left(L_{\mathcal{V}} \mathbf{1}_{\mathcal{S}}\right) \cong L_{f^{-1}} \mathcal{V} \mathbf{1}_{\mathcal{T}}
$$

Proof. The idempotent $\Gamma_{\mathcal{V}} \mathbf{1}_{\mathcal{S}}$ comes equipped with a morphism $\Gamma_{\mathcal{V}} \mathbf{1}_{\mathcal{S}} \stackrel{\varepsilon}{\longrightarrow} \mathbf{1}_{\mathcal{S}}$ giving the counit of the adjunction corresponding to the acyclisation functor with respect to $\Gamma_{\mathcal{V}} \mathcal{S}$. Applying the monoidal functor $F$ yields a morphism

$$
\varepsilon^{\prime}=\left(F\left(\Gamma_{\mathcal{V}} \mathbf{1}_{\mathcal{S}}\right) \stackrel{F(\varepsilon)}{\longrightarrow} F\left(\mathbf{1}_{\mathcal{S}}\right) \stackrel{\sim}{\longrightarrow} \mathbf{1}_{\mathcal{T}}\right) .
$$

Tensoring with this map yields a natural transformation, which we also denote by $\varepsilon^{\prime}$, with component $\varepsilon_{X}^{\prime}$ at $X \in \mathcal{T}$ given by $F\left(\Gamma_{\mathcal{V}} \mathbf{1}_{\mathcal{S}}\right) \otimes X \longrightarrow X$. We consider the full subcategory $\mathcal{M}$ of $\mathcal{T}$ defined as follows:

$$
\mathcal{M}=\left\{X \in \mathcal{T} \mid \varepsilon_{X}^{\prime} \text { is an isomorphism }\right\} .
$$

By naturality of $\varepsilon^{\prime}$ and its compatibility with coproducts and suspension, we deduce immediately that $\mathcal{M}$ is a localising subcategory of $\mathcal{T}$. Moreover, given $X \in \mathcal{M}$ and $Y \in \mathcal{T}$, commutativity of the square

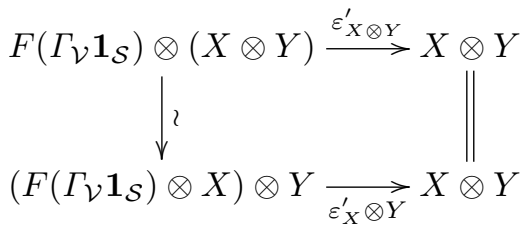

shows that $\mathcal{M}$ is a tensor ideal. Since $F$ is monoidal, we have $F\left(\Gamma_{\mathcal{V}} \mathcal{S}\right) \subseteq \mathcal{M}$ and so by the lemma $\Gamma_{f^{-1}} \mathcal{V} \mathcal{T} \subseteq \mathcal{M}$. Thus

$$
F\left(\Gamma_{\mathcal{V}} \mathbf{1}_{\mathcal{S}}\right) \cong \Gamma_{f^{-1} \mathcal{V}} \mathbf{1}_{\mathcal{T}} \otimes F\left(\Gamma_{\mathcal{V}} \mathbf{1}_{\mathcal{S}}\right) \cong \Gamma_{f^{-1} \mathcal{V}} \mathbf{1}_{\mathcal{T}}
$$

and the corresponding isomorphism for the localisation functors follows from uniqueness of localisation triangles.

Corollary 2.8. Let $\mathcal{V}$ be a Thomason subset of $\operatorname{Spc} \mathcal{S}^{c}$. Then

$\operatorname{supp} F\left(\Gamma_{\mathcal{V}} \mathbf{1}_{\mathcal{S}}\right)=f^{-1} \mathcal{V} \cap \operatorname{Vis} \mathcal{T}^{c} \quad$ and $\quad \operatorname{supp} F\left(L_{\mathcal{V}} \mathbf{1}_{\mathcal{S}}\right)=f^{-1}\left(\operatorname{Spc} \mathcal{S}^{c} \backslash \mathcal{V}\right) \cap \operatorname{Vis} \mathcal{T}^{c}$.

Proposition 2.9. Let $y$ be a visible point of $\operatorname{Spc} \mathcal{S}^{c}$. Then

$$
\operatorname{supp} F\left(\Gamma_{y} \mathbf{1}_{\mathcal{S}}\right)=f^{-1}(y) \cap \operatorname{Vis} \mathcal{T}^{c} .
$$

Moreover, $F\left(\Gamma_{y} \mathbf{1}_{\mathcal{S}}\right)$ is zero if and only if the fibre over $y$ is empty.

Proof. As $y$ is visible, we can find Thomason subsets $\mathcal{V}$ and $\mathcal{W}$ of $\operatorname{Spc} \mathcal{S}^{c}$ defining $\Gamma_{y} \mathbf{1}_{\mathcal{S}}$; i.e., our subsets satisfy $\mathcal{V} \backslash \mathcal{W}=\{y\}$ and $\Gamma_{y} \mathbf{1}_{\mathcal{S}}=\Gamma_{\mathcal{V}} \mathbf{1}_{\mathcal{S}} \otimes L_{\mathcal{W}} \mathbf{1}_{\mathcal{S}}$. Since $F$ is monoidal, we see, using Proposition 2.7,

$$
F\left(\Gamma_{y} \mathbf{1}_{\mathcal{S}}\right) \cong F\left(\Gamma_{\mathcal{V}} \mathbf{1}_{\mathcal{S}}\right) \otimes F\left(L_{\mathcal{W}} \mathbf{1}_{\mathcal{S}}\right) \cong \Gamma_{f^{-1}} \mathcal{V} \mathbf{1}_{\mathcal{T}} \otimes L_{f^{-1} \mathcal{W}} \mathbf{1}_{\mathcal{T}}
$$

Applying the above Corollary and [Ste13, Proposition 5.7(4)] then yields the desired 
equality

$$
\operatorname{supp} F\left(\Gamma_{y} \mathbf{1}_{\mathcal{S}}\right)=f^{-1} \mathcal{V} \cap\left(\operatorname{Spc} \mathcal{T}^{c} \backslash f^{-1} \mathcal{W}\right) \cap \operatorname{Vis} \mathcal{T}^{c}=f^{-1}(y) \cap \operatorname{Vis} \mathcal{T}^{c} .
$$

It follows that $F\left(\Gamma_{y} \mathbf{1}_{\mathcal{S}}\right)$ is non-zero iff the fibre over $y$ is non-empty. Indeed, $F\left(\Gamma_{y} \mathbf{1}_{\mathcal{S}}\right)$ is non-zero if and only if

$$
\Gamma_{f^{-1} \mathcal{V}} \mathbf{1}_{\mathcal{T}} \otimes L_{f^{-1} \mathcal{W}} \mathbf{1}_{\mathcal{T}} \neq 0 \text { i.e. } \quad f^{-1} \mathcal{V} \nsubseteq f^{-1} \mathcal{W}
$$

which occurs precisely when $f^{-1}(y) \neq \varnothing$.

Remark 2.10. Notice that in the special case $f^{-1}(y)=x$ the point $x$ is also visible and we have an isomorphism

$$
F\left(\Gamma_{y} \mathbf{1}_{\mathcal{S}}\right) \cong \Gamma_{x} \mathbf{1}_{\mathcal{T}}
$$

\subsection{Spectra and smashing localisations}

We now make some observations concerning the spectra of quotients by smashing ideals. Our point is to illustrate that there is a support-theoretic obstruction to being the right orthogonal of a smashing ideal; the results here form the basis for verifying the telescope conjecture for certain rings in Section 4.2.

Let $\mathcal{T}$ be a rigidly-compactly generated tensor triangulated category, let $\mathcal{S}$ be a smashing ideal of $\mathcal{T}$, and denote by $\mathcal{S}^{\perp}$ the right orthogonal of $\mathcal{S}$-i.e.,

$$
\mathcal{S}^{\perp}=\{B \in \mathcal{T} \mid \operatorname{Hom}(A, B)=0 \forall A \in \mathcal{S}\} .
$$

We recall there is an associated localisation sequence

$$
\mathcal{S} \underset{I^{!}}{\stackrel{I_{*}}{\rightleftarrows}} \mathcal{T} \underset{J_{*}}{\stackrel{J^{*}}{\rightleftarrows}} \mathcal{S}^{\perp},
$$

where $\mathcal{S}$ is a localising tensor ideal of $\mathcal{T}$ and $I^{!}$preserves coproducts. It follows that $J_{*}$ also preserves coproducts and $\mathcal{S}^{\perp}$ is also a localising tensor ideal of $\mathcal{T}$. The reader may consult [BF11] for further details.

We thus see that $\mathcal{S}^{\perp}$ is a compactly generated tensor triangulated category and $J^{*}$ is an exact monoidal functor that sends compact objects to compact objects. Moreover, there are tensor idempotents $\Gamma_{\mathcal{S}} \mathbf{1}$ and $L_{\mathcal{S}} \mathbf{1}$ such that

$$
\Gamma_{\mathcal{S}} \mathbf{1} \otimes(-) \cong I_{*} I^{!} \text {and } L_{\mathcal{S}} \mathbf{1} \otimes(-) \cong J_{*} J^{*}
$$

Let us denote the restriction of $J^{*}$ to compacts by $j^{*}: \mathcal{T}^{c} \longrightarrow\left(\mathcal{S}^{\perp}\right)^{c}$, and let us denote the associated spectral map by

$$
j: \operatorname{Spc}\left(\mathcal{S}^{\perp}\right)^{c} \longrightarrow \operatorname{Spc} \mathcal{T}^{c} .
$$

By [Kra05, Theorem 11.1] the functor $j^{*}$ is essentially surjective up to direct summands. As an immediate consequence we deduce the following lemma.

Lemma 2.11. The map $j: \operatorname{Spc}\left(\mathcal{S}^{\perp}\right)^{c} \longrightarrow \operatorname{Spc} \mathcal{T}^{c}$ is injective.

Proof. This is essentially [Bal05, Corollary 3.8]-weakening essential surjectivity to essential surjectivity up to summands does no harm; one just needs to close under summands as well as isomorphisms in the argument given there. 
In order to simplify the discussion, and since it is enough for our intended application, we will assume every point of $\operatorname{Spc} \mathcal{T}^{c}$ is visible. Combining the last lemma with Remark 2.10, we see every point of $\operatorname{Spc}\left(\mathcal{S}^{\perp}\right)^{c}$ is also visible.

We now identify the image of the map $j$ as a set.

Lemma 2.12. There is an equality of sets

$$
\operatorname{im} j=\operatorname{supp} \mathcal{S}^{\perp} .
$$

Proof. Let $x$ be a point of $\operatorname{Spc} \mathcal{T}^{c}$. We have $x \in \operatorname{supp} \mathcal{S}^{\perp}$ if and only if there is an $X \in \mathcal{S}^{\perp}$ such that $\Gamma_{x} \mathbf{1}_{\mathcal{T}} \otimes X$ is non-zero. As $Y \cong L_{\mathcal{S}} \mathbf{1}_{\mathcal{T}} \otimes Y$ for all $Y \in \mathcal{S}^{\perp}$, we see there exists such an $X$ if and only if $L_{\mathcal{S}} \mathbf{1}_{\mathcal{T}} \otimes \Gamma_{x} \mathbf{1}_{\mathcal{T}}$ is non-zero-i.e., $J^{*} \Gamma_{x} \mathbf{1}_{\mathcal{T}} \neq 0$. We proved in Proposition 2.9 that $J^{*} \Gamma_{x} \mathbf{1}_{\mathcal{T}} \neq 0$ if and only if $j^{-1}(x)$ is non-empty.

Tracing through this chain of equivalent statements, we find that $x \in \operatorname{supp} \mathcal{S}^{\perp}$ if and only if there is a $y \in \operatorname{Spc}\left(\mathcal{S}^{\perp}\right)^{c}$ such that $j(y)=x$, which is precisely what we have claimed.

Lemma 2.13. The map $j$, viewed as a morphism to $\operatorname{supp} \mathcal{S}^{\perp}$ with the subspace topology, is closed and is thus a homeomorphism.

Proof. It is, of course, sufficient to check $j$ is closed on a basis of closed subsets for $\operatorname{Spc}\left(\mathcal{S}^{\perp}\right)^{c}$, and so we may reduce to considering the supports of compact objects. Given $a \in\left(\mathcal{S}^{\perp}\right)^{c}$, we will show there is a $b \in \mathcal{T}^{c}$ satisfying

$$
j(\operatorname{supp} a)=\operatorname{supp} b \cap \operatorname{supp} \mathcal{S}^{\perp} .
$$

We may assume by replacing $a$, if necessary, by $a \oplus \Sigma a$ that there is a $b \in \mathcal{T}^{c}$ with $j^{*} b=a$ (using [Nee01, Proposition 4.5.11]); we note making this replacement does not change the support.

We then just need to observe the following series of equalities:

$$
\begin{aligned}
j(\operatorname{supp} a) & =\left\{j(\mathcal{P}) \mid \mathcal{P} \in \operatorname{Spc}\left(\mathcal{S}^{\perp}\right)^{c} \text { and } a \notin \mathcal{P}\right\} \\
& =\left\{\left(j^{*}\right)^{-1} \mathcal{P} \mid \mathcal{P} \in \operatorname{Spc}\left(\mathcal{S}^{\perp}\right)^{c} \text { and } j^{*} b \notin \mathcal{P}\right\} \\
& =\left\{\left(j^{*}\right)^{-1} \mathcal{P} \mid \mathcal{P} \in \operatorname{Spc}\left(\mathcal{S}^{\perp}\right)^{c} \text { and } b \notin\left(j^{*}\right)^{-1} \mathcal{P}\right\} \\
& =\left\{\mathcal{Q} \in \operatorname{supp} \mathcal{S}^{\perp} \mid b \notin \mathcal{Q}\right\} \\
& =\operatorname{supp} b \cap \operatorname{supp} \mathcal{S}^{\perp} .
\end{aligned}
$$

The main result of this section is stated in terms of the constructible topology on a spectral space (see Definition 2.1).

Proposition 2.14. Let $\mathcal{T}$ be a rigidly-compactly generated tensor triangulated category such that every point of $\operatorname{Spc} \mathcal{T}^{c}$ is visible. Given any smashing tensor ideal $\mathcal{S}$ of $\mathcal{T}$, the subset $\operatorname{supp} \mathcal{S}^{\perp}$ is proconstructible in $\operatorname{Spc} \mathcal{T}^{c}$.

Proof. By the last lemma we know $\operatorname{supp} \mathcal{S}^{\perp}$ with the subspace topology is homeomorphic to $\operatorname{Spc}\left(\mathcal{S}^{\perp}\right)^{c}$. Thus $\operatorname{supp} \mathcal{S}^{\perp}$ is a spectral space and the inclusion of $\operatorname{supp} \mathcal{S}^{\perp}$ 
into $\operatorname{Spc} \mathcal{T}^{c}$ is spectral. The inclusion thus induces a spectral map

$$
\left(\operatorname{supp} \mathcal{S}^{\perp}\right)^{\mathrm{con}} \longrightarrow\left(\mathrm{Spc} \mathcal{T}^{c}\right)^{\mathrm{con}}
$$

which is again a homeomorphism onto its image. As $\left(\operatorname{Spc} \mathcal{T}^{c}\right)^{\text {con }}$ is Hausdorff and $\left(\operatorname{supp} \mathcal{S}^{\perp}\right)^{\text {con }}$ is a quasi-compact subset, it is closed in $\left(\operatorname{Spc} \mathcal{T}^{c}\right)^{\text {con }}$; i.e., it is proconstructible in $\operatorname{Spc} \mathcal{T}^{c}$.

\section{Preliminaries on absolutely flat rings}

Before beginning our study of derived categories it seems prudent to provide some brief recollections on the class of rings with which we will be concerned. Throughout, all of our rings are assumed to be commutative and unital.

Definition 3.1. Let $R$ be a commutative ring with unit. We say $R$ is absolutely flat (also known as von Neumann regular) if for every $r \in R$ there exists some $x \in R$ satisfying

$$
r=r^{2} x \text {. }
$$

Absolutely flat rings arise naturally when studying the constructible topology on spectral spaces. As we shall see below, one can functorially associate to a ring $S$ an absolutely flat ring $R$ with the property that $\operatorname{Spec} R$ is naturally homeomorphic to Spec $S$ endowed with the constructible topology.

From now on $R$ denotes a commutative absolutely flat ring. We will assume $R$ is not noetherian. One deduces easily from the commutativity of $R$ that for every $r \in R$ there is a unique $x \in R$ such that $r=r^{2} x$ and $x=x^{2} r$. We call $x$ the weak inverse of $r$.

We now collect some standard characterisations of commutative absolutely flat rings; we will, in general, use these properties without reference to the proposition.

Proposition 3.2. For a ring $R$ the following are equivalent:

(i) $R$ is absolutely flat;

(ii) $R$ is reduced and has Krull dimension 0 ;

(iii) every localization of $R$ at a prime ideal is a field;

(iv) $R$ is a subring of a product of fields, namely,

$$
R \subseteq \prod_{\mathfrak{p} \in \operatorname{Spec} R} k(\mathfrak{p})=: R^{\prime},
$$

where $k(\mathfrak{p})$ is the residue field at $\mathfrak{p}$, and $R$ is closed under weak inverses in $R^{\prime}$;

$(v)$ every simple $R$-module is injective;

(vi) every $R$-module is flat.

Proof. The equivalence of $(i),(i i)$, and (vi) together with the implication $(i)$ implies (iii) can be found, for instance, in [Sta14, Tag 092F]. The equivalence of $(i)$ and $(v)$ is a result of Kaplansky; for a proof the reader can consult [MV73]. Finally, the equivalence of $(i)$ and $(i v)$ is straightforward. 
The spectrum of $R$, Spec $R$, is a zero-dimensional, Hausdorff, totally disconnected spectral space. We wish to say a little more about it. The first two lemmas are essentially trivialities and are in fact valid for the constructible topology on any spectral space.

Lemma 3.3. A subset $Z \subseteq \operatorname{Spec} R$ is closed if and only if it quasi-compact.

Proof. This is immediate from the fact that $\operatorname{Spec} R$ is Hausdorff.

Lemma 3.4. A subset $V \subseteq \operatorname{Spec} R$ is Thomason if and only if it is open.

Proof. Let $U$ be a quasi-compact open subset of Spec $R$. Then, by the last lemma, $U$ is also closed, and its complement, by virtue of being closed, is also quasi-compact; i.e., both $U$ and its complement are closed Thomason subsets. As Spec $R$ is spectral, the subset $V$ is open if and only if it is a union of quasi-compact open subsets of Spec $R$. But we have just shown this is precisely the same thing as being a union of closed subsets with quasi-compact complement, i.e., as being Thomason.

Lemma 3.5. The spectrum of $R$ is infinite; i.e., $|\operatorname{Spec} R| \geqslant \aleph_{0}$.

Proof. Suppose $|\operatorname{Spec} R|<\aleph_{0}$ so $R$ has finitely many prime ideals $\mathfrak{p}_{1}, \ldots, \mathfrak{p}_{n}$. Then $R$ is, by Proposition 3.2, a subring of $S=k\left(\mathfrak{p}_{1}\right) \times \cdots \times k\left(\mathfrak{p}_{n}\right)$. Clearly $S$ is noetherian and module finite over $R$, so by the Eakin-Nagata theorem $R$ is noetherian (hence isomorphic to $S$ ), which is a contradiction.

Lemma 3.6. The spectrum of $R$ is not a noetherian topological space.

Proof. By the last lemma $\operatorname{Spec} R$ has infinitely many points. Thus, since it is quasicompact, $\operatorname{Spec} R$ cannot be discrete. So there is a point $\mathfrak{p} \in \operatorname{Spec} R$ with $\{\mathfrak{p}\}$ not open. Thus Spec $R \backslash\{\mathfrak{p}\}$ is open but not closed and hence not quasi-compact by Lemma 3.3. This demonstrates that $\operatorname{Spec} R$ is not noetherian.

Remark 3.7. The proof of the lemma exhibits a point of $\operatorname{Spec} R$ that is not a Thomason subset.

We conclude by reminding the reader that one can functorially associate to any commutative ring $S$ an absolutely flat ring $S^{\text {abs }}$. Let CRing denote the category of commutative unital rings, and let CRing ${ }^{\text {abs }}$ denote the full subcategory of absolutely flat rings.

Theorem 3.8 ([Oli67a, Proposition 5]). The inclusion CRing $^{\text {abs }} \longrightarrow$ CRing admits a left adjoint

$$
(-)^{\text {abs }} \text { : CRing } \longrightarrow \text { CRing }^{\text {abs }} \text {. }
$$

Given a ring $S$, the unit of adjunction $\eta: S \longrightarrow S^{\text {abs }}$ induces a bijection of sets

$$
\text { Spec } S^{\text {abs }} \longrightarrow \operatorname{Spec} S
$$

and isomorphisms $\left(S^{\text {abs }}\right)_{P} \cong k\left(\eta^{-1} P\right)$ for all $P \in \operatorname{Spec} S^{\text {abs }}$. Furthermore, there is a homeomorphism

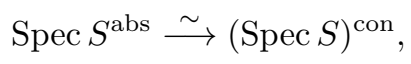

i.e., $S^{\text {abs }}$ realises the constructible topology on $\operatorname{Spec} S$ (see Definition 2.1). 
Given a ring $S$, one can explicitly construct $S^{\text {abs }}$ as

$$
S^{\mathrm{abs}}=S\left[x_{s} \mid s \in S\right] /\left(s^{2} x_{s}-s, x_{s}^{2} s-x_{s} \mid s \in S\right) .
$$

Example 3.9. Suppose we take for $S$ the ring $\mathbb{Z}$ of integers. Then it is an entertaining exercise to check that, using the above recipe, one can describe $R=\mathbb{Z}^{\text {abs }}$ as the subring of the product of the residue fields of $\mathbb{Z}$ defined by certain congruences. To be precise, $S$ is the subring of $\mathbb{Q} \times \prod_{p} \mathbb{F}_{p}$, where $p$ runs over all primes, consisting of those sequences $\left\{a_{\mathbb{Q}}, a_{\mathbb{F}_{p}}\right\}_{p}$ such that there is an $N \in \mathbb{Q}$ satisfying $N a_{\mathbb{Q}} \in \mathbb{Z}$ and $a_{\mathbb{Q}} \equiv a_{\mathbb{F}_{p}}$ modulo $p$ for all $p \nmid N$.

\section{Derived categories of absolutely flat rings}

Throughout this section $R$ is again a non-noetherian absolutely flat ring. As usual, $\mathrm{D}(R)$ denotes the unbounded derived category of $R$, and $\mathrm{D}^{\text {perf }}$ denotes $(R)$ the full subcategory of perfect complexes. For a prime ideal $\mathfrak{p}$ of $R$ we denote by $k(\mathfrak{p})$ the residue field at $\mathfrak{p}$. We recall that $\mathrm{D}(R)$ is a rigidly-compactly generated tensor triangulated category with a monoidal model (i.e., there is a symmetric monoidal model category whose homotopy category is $\mathrm{D}(R))$, the subcategory $\mathrm{D}^{\text {perf }}(R)$ is the full subcategory of compact objects in $\mathrm{D}(R)$, and by a theorem of Thomason [Tho97]

$$
\operatorname{Spc} \mathrm{D}^{\text {perf }}(R) \cong \operatorname{Spec} R \text {. }
$$

For $X \in \mathrm{D}(R)$ we set

$$
\operatorname{supph} X=\{\mathfrak{p} \in \operatorname{Spec} R \mid X \otimes k(\mathfrak{p}) \neq 0\} .
$$

Since $R$ is absolutely flat, there is no need to derive the tensor product, although, in any case, $R_{\mathfrak{p}} \cong k(\mathfrak{p})$. Thus $\operatorname{supph} X$ is the set of primes $\mathfrak{p}$ such that $X_{\mathfrak{p}}$ is not acyclic. This observation immediately yields the following lemma.

Lemma 4.1. An object $X$ of $\mathrm{D}(R)$ is zero if and only if $\operatorname{supph} X=\varnothing-i . e$., if and only if $X \otimes k(\mathfrak{p}) \cong 0$ for all $\mathfrak{p} \in \operatorname{Spec} R$.

We now connect this support with Balmer's support on the compact objects and with the corresponding Rickard idempotents.

Lemma 4.2. For every $\mathfrak{p} \in \operatorname{Spec} R$ the subset $\mathcal{Z}(\mathfrak{p})=\operatorname{Spec} R \backslash\{\mathfrak{p}\}$ is Thomason and the corresponding Rickard idempotent $L_{\mathcal{Z}(\mathfrak{p})} R$ is canonically isomorphic to $k(\mathfrak{p})$. The resulting localisation corresponds to the fully faithful inclusion of $\mathrm{D}\left(R_{\mathfrak{p}}\right)=\mathrm{D}(k(\mathfrak{p}))$ in $\mathrm{D}(R)$.

Proof. It is standard that $\mathcal{Z}(\mathfrak{p})$ is Thomason (it is also immediate from Lemma 3.4). For the second claim consider the adjunction

$$
\mathrm{D}(R) \stackrel{R_{\mathfrak{p}} \otimes-}{\rightleftarrows} \mathrm{D}\left(R_{\mathfrak{p}}\right)
$$

given by base change and restriction of scalars. As the restriction functor is fully faithful, this is in fact a localisation, and one easily identifies the kernel of $R_{\mathfrak{p}} \otimes-$ with $\Gamma_{\mathcal{Z}(\mathfrak{p})} \mathrm{D}(R)$. Thus we have an equivalence $D\left(R_{\mathfrak{p}}\right) \cong L_{\mathcal{Z}(\mathfrak{p})} \mathrm{D}(R)$, which in turn yields an isomorphism $L_{\mathcal{Z}(\mathfrak{p})} R \cong R_{\mathfrak{p}}=k(\mathfrak{p})$. 
Thus, even though there are points that are not Thomason subsets, every point of $\operatorname{Spc} D^{\text {perf }}(R)$ is visible via $\operatorname{Spec} R \backslash \mathcal{Z}(\mathfrak{p})=\{\mathfrak{p}\}$. So we can associate to every point $\mathfrak{p}$ a coproduct-preserving endofunctor $\Gamma_{\mathfrak{p}}$. Here $\Gamma_{\mathfrak{p}}$ is just (yet) another notation for $k(\mathfrak{p}) \otimes(-)$,

$$
\Gamma_{\mathfrak{p}} R=\Gamma_{\text {Spec } R} L_{\mathcal{Z}(\mathfrak{p})} R \cong k(\mathfrak{p}),
$$

but it is a conceptually helpful one. We see the support theory defined as in [BF11], which we have studied in Section 2, agrees with the homological support supph. From this point onward we will just write $\operatorname{supp} X$ for these coinciding notions of the support of an object $X$ of $\mathrm{D}(R)$.

Remark 4.3. We feel it is worth reiterating that this example shows there can exist points whose closure is not Thomason but which are visible and thus have associated idempotents. This shows the definition of visible point, as given in [Ste13] (and here in Section 2), is more general than the one in [BF11].

We now investigate $\mathrm{D}(R)$ and, in particular, this support theory with a view toward comparison with rigidly-compactly generated tensor triangulated categories whose compact objects have noetherian spectrum (as considered in [BIK11] and [Ste13]). More specifically, we consider the analogues of the results of Neeman [Nee92], namely, the classification of localising subcategories and the telescope conjecture. It turns out that there is a dichotomy - the derived category either behaves very differently (and somewhat mysteriously) or behaves exactly as in the noetherian case where it is possible to completely understand the localising subcategories.

We begin with a few general statements that are essentially standard.

Lemma 4.4. For each $\mathfrak{p} \in \operatorname{Spec} R$ the localising subcategory $\langle k(\mathfrak{p})\rangle$ is minimal in $\mathrm{D}(R)$, i.e., it has no proper non-trivial localising subcategories.

Proof. As in Lemma 4.2, we can identify $\langle k(\mathfrak{p})\rangle$ with $\mathrm{D}(k(\mathfrak{p}))$. Since $k(\mathfrak{p})$ is a field, every object of $\mathrm{D}(k(\mathfrak{p}))$ is a sum of suspensions of $k(\mathfrak{p})$. As localising subcategories are closed under splitting idempotents, we see that any non-zero localising subcategory of $\mathrm{D}(k(\mathfrak{p}))$ must be the whole category.

The next lemma can be found, in a slightly more general form, as [BN93, Lemma 2.17] and already appears in the work of Foxby [Fox79].

Lemma 4.5. Let $X$ be an object of $\mathrm{D}(R)$. Then for $\mathfrak{p} \in \operatorname{Spec} R$ the object $X \otimes k(\mathfrak{p})$ is isomorphic to a coproduct of suspensions of $k(\mathfrak{p})$.

From these two lemmas we deduce the following result.

Lemma 4.6. There is a bijection between $\operatorname{Spec} R$ and the minimal non-zero localising subcategories of $\mathrm{D}(R)$ given by sending $\mathfrak{p}$ to the localising subcategory $\langle k(\mathfrak{p})\rangle$ of $\mathrm{D}(R)$.

Proof. Lemma 4.4 associates a non-zero minimal localising subcategory to each $\mathfrak{p} \in$ $\operatorname{Spec} R$. It remains to show that these are precisely the non-zero minimal localising subcategories. Suppose then that $\mathcal{L}$ is non-zero, localising, and minimal in $\mathrm{D}(R)$. Let $X \in \mathcal{L}$ be a non-zero object. Since, by Lemma 4.1, the support detects vanishing of objects, there is a $\mathfrak{p} \in \operatorname{Spec} R$ with $X \otimes k(\mathfrak{p})$ non-zero. As every localising subcategory of $\mathrm{D}(R)$ is tensor closed, Lemma 4.5 implies $k(\mathfrak{p}) \in \mathcal{L}$ and hence $\langle k(\mathfrak{p})\rangle \subseteq \mathcal{L}$. Minimality of $\mathcal{L}$ then forces this to be an equality. 


\subsection{Rings behaving badly}

So far this actually seems very promising: the reader might hope that, since we have a seemingly well-behaved support theory and know the minimal localising subcategories, we can describe the collection of all localising subcategories in terms of Spec $R$. The next result should extinguish this hope, at least at our current level of generality. The reader who prefers the good news first should skip to Section 4.2.

Definition 4.7. Let $S$ be a ring (not necessarily absolutely flat). We say $S$ is semiartinian if every non-zero homomorphic image of $S$, in the category of $S$-modules, contains a simple submodule.

An $S$-module $M$ is said to be superdecomposable if it admits no non-zero indecomposable direct summands.

Theorem 4.8. Let $R$ be an absolutely flat ring that is not semi-artinian. Then the residue fields do not generate $\mathrm{D}(R)$-i.e,

$$
\langle k(\mathfrak{p}) \mid \mathfrak{p} \in \operatorname{Spec} R\rangle \subsetneq \mathrm{D}(R) .
$$

Proof. By a result of Trlifaj [Tr196] there exists a superdecomposable injective $R$ module $E$. We claim $E$ is right orthogonal to each $k(\mathfrak{p})$. As $E$ is injective, the only other possibility is that $\operatorname{Hom}(k(\mathfrak{p}), E)$ is non-zero. But if there were a non-zero map $k(\mathfrak{p}) \longrightarrow E$, then, since the residue fields are simple, it would have to be a monomorphism. The module $k(\mathfrak{p})$ is injective (see Proposition 3.2), so this morphism would then split and exhibit $k(\mathfrak{p})$ as an indecomposable direct summand of $E$, contradicting the superdecomposability of $E$. Thus $\operatorname{Hom}\left(k(\mathfrak{p}), \Sigma^{i} E\right)$ vanishes for all $i \in \mathbb{Z}$; i.e., $E$ lies in the right orthogonal of $\langle k(\mathfrak{p}) \mid \mathfrak{p} \in \operatorname{Spec} R\rangle$. In particular, $E$ cannot lie in $\langle k(\mathfrak{p}) \mid \mathfrak{p} \in \operatorname{Spec} R\rangle$, proving this subcategory is properly contained in $\mathrm{D}(R)$ as claimed.

Combining this with Lemma 4.6, we see, when $R$ is not semi-artinian, the minimal localising subcategories of $\mathrm{D}(R)$ do not generate it. In other words, the local-to-global principle (see [Ste13, Definition 6.1]) fails.

There are several conjectures, originating from the study of the stable homotopy category, concerning the lattice of localising subcategories. These conjectures make sense in any rigidly-compactly generated tensor triangulated category, and trying to resolve them in other settings can hopefully provide intuition for the original conjectures. The theorem allows us to give counterexamples to the derived category analogues of two such conjectures.

Definition 4.9. A localising subcategory $\mathcal{L}$ of $\mathrm{D}(R)$ is a Bousfield class if there is an $X \in \mathrm{D}(R)$ such that

$$
\mathcal{L}=\{Y \in \mathrm{D}(R) \mid X \otimes Y \cong 0\}=\operatorname{ker}(X \otimes(-)) .
$$

The localising subcategory $\mathcal{L}$ is a cohomological Bousfield class if there is an $X \in \mathrm{D}(R)$ such that

$$
\mathcal{L}=\left\{Y \in \mathrm{D}(R) \mid \operatorname{Hom}\left(Y, \Sigma^{i} X\right)=0 \quad \forall i \in \mathbb{Z}\right\}={ }^{\perp} X .
$$

One calls $\operatorname{ker}(X \otimes(-))$ the Bousfield class of $X$ and ${ }^{\perp} X$ the cohomological Bousfield class of $X$. 
Corollary 4.10. Let $R$ be an absolutely flat ring that is not semi-artinian. Then $\mathrm{D}(R)$ admits a localising subcategory that is not a Bousfield class.

Proof. By the theorem $\mathcal{L}=\langle k(\mathfrak{p}) \mid \mathfrak{p} \in \operatorname{Spec} R\rangle$ is a proper localising subcategory of $\mathrm{D}(R)$. In particular, it is not the Bousfield class of 0 . As the support detects vanishing by Lemma 4.1, the Bousfield class of any non-zero $X \in \mathrm{D}(R)$ fails to contain some $k(\mathfrak{p})$. Thus $\mathcal{L}$ cannot be a Bousfield class.

Remark 4.11. This gives a counterexample to the analogue of [HP99, Conjecture 9.1] for the derived category of a ring.

Corollary 4.12. Let $R$ be an absolutely flat ring that is not semi-artinian. Then $\mathrm{D}(R)$ admits a cohomological Bousfield class that is not a Bousfield class.

Proof. As in the proof of the theorem there is, by [Tr196], a superdecomposable injective $E$ and

$$
\langle k(\mathfrak{p}) \mid \mathfrak{p} \in \operatorname{Spec} R\rangle \subseteq{ }^{\perp} E .
$$

It is clear that ${ }^{\perp} E$ is a proper localising subcategory of $\mathrm{D}(R)$ and so, by Lemma 4.1, ${ }^{\perp} E$ cannot be a Bousfield class as in the argument proving the last Corollary.

Remark 4.13. This gives a counterexample to the analogue of [Hov95, Conjecture 1.2] for the derived category of a ring.

Example 4.14. Let $\Lambda$ be an infinite index set, and for each $\lambda \in \Lambda$ let $k_{\lambda}$ be a field. The ring

$$
R=\prod_{\lambda \in \Lambda} k_{\lambda}
$$

is absolutely flat. By [CS96, Lemma 1] it is not semi-artinian, and so the theorem and both corollaries apply. In particular, the non-zero minimal localising subcategories of $\mathrm{D}(R)$ do not generate $\mathrm{D}(R)$.

\subsection{Some good behaviour}

We now use the abstract nonsense of Section 2 to show that for a certain class of absolutely flat rings one does not observe the same interesting behaviour as in the last section.

Let $S$ be a commutative unital ring such that Spec $S$ is a noetherian topological space - for instance, $S$ could be a noetherian ring. Since $\operatorname{Spec} S$ is noetherian, every point of $\operatorname{Spc} D^{\text {perf }}(S) \cong \operatorname{Spec} S$ is visible. Set $R=S^{\text {abs }}$, and denote by $f: S \longrightarrow R$ the canonical map (see Theorem 3.8 for details). Our first aim is to prove that Spec $S$ being noetherian implies $\mathrm{D}(R)$ is generated by the residue fields of $R$.

The first lemma we need is just an application of our general nonsense to the exact, monoidal, and compact object preserving functor $\mathbf{L} f^{*}: \mathrm{D}(S) \longrightarrow \mathrm{D}(R)$ given by taking the left derived functor of $f^{*}=R \otimes_{S}(-)$.

Lemma 4.15. Let $\mathfrak{p} \in \operatorname{Spec} S$, and denote by $P$ the unique point of $\operatorname{Spec} R$ such that $f^{-1} P=\mathfrak{p}$. Then

$$
\mathbf{L} f^{*}\left(\Gamma_{\mathfrak{p}} S\right) \cong k(P)
$$


Proof. As in the statement let $\mathfrak{p}$ be a point of $\operatorname{Spec} S$, and let $P$ be the corresponding point of $\operatorname{Spec} R$. We are in the situation of Section 2, so we may apply Proposition 2.7 to obtain isomorphisms

$$
\begin{aligned}
\mathbf{L} f^{*}\left(\Gamma_{\mathfrak{p}} S\right) & =\mathbf{L} f^{*}\left(\Gamma_{\mathcal{V}(\mathfrak{p})} S \otimes L_{\mathcal{Z}(\mathfrak{p})} S\right) \\
& \cong \mathbf{L} f^{*}\left(\Gamma_{\mathcal{V}(\mathfrak{p})} S\right) \otimes \mathbf{L} f^{*}\left(L_{\mathcal{Z}(\mathfrak{p})} S\right) \\
& \cong \Gamma_{f^{-1} \mathcal{V}(\mathfrak{p})} R \otimes L_{f^{-1} \mathcal{Z}(\mathfrak{p})} R .
\end{aligned}
$$

As $f$ is a bijection, we have

$$
f^{-1} \mathcal{V}(\mathfrak{p}) \backslash f^{-1} \mathcal{Z}(\mathfrak{p})=\{P\}
$$

This yields isomorphisms

$$
\Gamma_{f^{-1} \mathcal{V}(\mathfrak{p})} R \otimes L_{f^{-1} \mathcal{Z}(\mathfrak{p})} R \cong \Gamma_{P} R \cong k(P),
$$

the first by the independence of $\Gamma_{P} R$ on the subsets used to define it (see [BF11, Corollary 7.5]) and the second by the observation of equation (1), completing the proof.

Remark 4.16. Assuming the spectrum of $S$ is noetherian is not essential for the lemma provided one corrects the statement by considering only visible points (i.e., the points for which the statement makes sense).

It seems appropriate at this point to briefly remark on actions of tensor triangulated categories as studied in [Ste13] since we will use some of the language of tensor actions in the sequel. Let $(\mathcal{T}, \otimes, \mathbf{1})$ be a rigidly-compactly generated tensor triangulated category, and let $\mathcal{K}$ be a compactly generated triangulated category. A left action of $\mathcal{T}$ on $\mathcal{K}$ is a functor

$$
*: \mathcal{T} \times \mathcal{K} \longrightarrow \mathcal{K}
$$

that is exact in each variable, i.e., for all $X \in \mathcal{T}$ and $A \in \mathcal{K}$ the functors $X *(-)$ and $(-) * A$ are exact, together with natural isomorphisms

$$
a_{X, Y, A}:(X \otimes Y) * A \stackrel{\sim}{\longrightarrow} X *(Y * A)
$$

and

$$
l_{A}: 1 * A \stackrel{\sim}{\longrightarrow} A
$$

for all $X, Y \in \mathcal{T}, A \in \mathcal{K}$, compatible with the exactness of $(-) *(-)$ and satisfying associativity and unitality constraints. For example $\mathcal{T}$ acts on itself in a natural way via its monoidal structure.

In such a situation one has a theory of supports for objects of $\mathcal{K}$ with values in $\operatorname{Spc} \mathcal{T}$, the spectrum of $\mathcal{T}$. More precisely, one defines for $A \in \mathcal{K}$

$$
\operatorname{supp} A=\left\{x \in \operatorname{Spc} \mathcal{T} \mid \Gamma_{x} \mathbf{1} * A \neq 0\right\},
$$

where $\Gamma_{x} \mathbf{1}_{\mathcal{T}}$ is as in Section 2.1. The hope is that, provided one has a good understanding of $\mathcal{T}$, one can use this notion of support to obtain information on the localising subcategories of $\mathcal{K}$. The main technical tool in this regard is the local-to-global principle; as the name suggests, it allows one to reduce the classification of localising subcategories of $\mathcal{K}$ to studying localising subcategories in the essential images of 
tensoring with the $\Gamma_{x} \mathbf{1}$. We say the local-to-global principle holds for the action $*$ of $\mathcal{T}$ on $\mathcal{K}$ if for each $A$ in $\mathcal{K}$

$$
\langle A\rangle_{*}=\left\langle\Gamma_{x} A \mid x \in \operatorname{Spc} \mathcal{T}^{c}\right\rangle_{*},
$$

where $\langle A\rangle_{*}$ and $\left\langle\Gamma_{x} A \mid x \in \operatorname{Spc} \mathcal{T}^{c}\right\rangle_{*}$ are the smallest localizing subcategories of $\mathcal{K}$ containing $A$ or the $\Gamma_{x} A$, respectively, and closed under the action of $\mathcal{T}$. If $\mathcal{T}$ is generated by $\mathbf{1}$, then any localising subcategory of $\mathcal{K}$ is automatically closed under the action ([Ste13, Lemma 3.13]), and so one can just take the smallest localising subcategories containing the given sets of objects.

While we are giving definitions, let us take this opportunity to clarify what we mean by the telescope conjecture and introduce a version relative to an action.

Definition 4.17. Let $\mathcal{K}$ be a compactly generated triangulated category. We say the telescope conjecture holds for $\mathcal{K}$ if every smashing subcategory of $\mathcal{K}$ is generated by objects that are compact in $\mathcal{K}$.

Now suppose $\mathcal{T}$ is a rigidly-compactly generated tensor triangulated category that acts on $\mathcal{K}$. We say the relative telescope conjecture holds for $\mathcal{K}$ with respect to the action of $\mathcal{T}$ if every smashing $\mathcal{S} \subseteq \mathcal{K}$ that is closed under the action of $\mathcal{T}$ is generated by compact objects of $\mathcal{K}$.

Continuing on after this aside, we now we invoke the hypothesis that $\operatorname{Spec} S$ is noetherian. As we have mentioned earlier, $\mathrm{D}(S)$ is always a rigidly-compactly generated tensor triangulated category with a monoidal model, and, by a result of Thomason [Tho97], Spc $\mathrm{D}^{\text {perf }}(S)$ is canonically homeomorphic to $\operatorname{Spec} S$. Thus, as $\operatorname{Spec} S$ is noetherian, we may apply [Ste13, Theorem 6.9]: the local-to-global principle holds for the action of $\mathrm{D}(S)$ on itself and the support detects vanishing. In particular, we have equalities

$$
\mathrm{D}(S)=\langle S\rangle=\left\langle\Gamma_{\mathfrak{p}} S \mid \mathfrak{p} \in \operatorname{Spec} S\right\rangle .
$$

Combined with the lemma, this has the following consequence.

Proposition 4.18. The derived category $\mathrm{D}(R)$ is generated by the residue fields of $R$-i.e.,

$$
\mathrm{D}(R)=\langle k(P) \mid P \in \operatorname{Spec} R\rangle .
$$

Proof. By (2) $S$ can be built from the $\Gamma_{\mathfrak{p}} S$, so by a standard argument

$$
R \cong \mathbf{L} f^{*} S \in\left\langle\mathbf{L} f^{*}\left(\Gamma_{\mathfrak{p}} S\right) \mid \mathfrak{p} \in \operatorname{Spec} S\right\rangle .
$$

Applying the last lemma, this says $R$ lies in the localising subcategory $\langle k(P)| P \in$ Spec $R\rangle$. This proves the proposition as any localising subcategory of $\mathrm{D}(R)$ containing $R$ must be all of $\mathrm{D}(R)$.

Corollary 4.19. If $S$ is a ring with noetherian spectrum, then $R=S^{\text {abs }}$ is semiartinian.

Proof. We have proved in Theorem 4.8 that if $R$ is not semi-artinian then the residue fields do not generate $\mathrm{D}(R)$, so, given the proposition, $R$ had better be semi-artinian. 
In fact, we could have proved the proposition by more general methods. The monoidal functor $\mathbf{L} f^{*}$ furnishes us with an action of $\mathrm{D}(S)$ on $\mathrm{D}(R)$ as defined in [Ste13]. As we have noted above [Ste13, Theorem 6.9] guarantees this action satisfies the local-to-global principle. Furthermore, we have shown in Lemma 4.15 that for $\mathfrak{p} \in \operatorname{Spec} S \cong \operatorname{Spc} \mathrm{D}^{\text {perf }}(S)$ the object $\Gamma_{\mathfrak{p}} S$ acts on $\mathrm{D}(R)$ as $k(P)$ where $P$ is the corresponding point of Spec $R$. Thus the support obtained from the action of $\mathrm{D}(S)$ on $\mathrm{D}(R)$ agrees with the homological support on $\mathrm{D}(R)$. This essentially proves the following lemma.

Lemma 4.20. The homological support on $\mathrm{D}(R)$ satisfies the local-to-global principle-i.e., for $A \in \mathrm{D}(R)$

$$
\langle A\rangle=\langle k(P) \mid P \in \operatorname{supp} A\rangle .
$$

Remark 4.21. This demonstrates that, at least in special situations, one can use the action of some associated tensor triangulated category, for which the local-to-global principle is known, in order to obtain the local-to-global principle for the category of interest.

Remark 4.22. At this juncture it is worth making explicit that we are not in an entirely satisfactory situation: we have shown that the local-to-global principle does not hold for non-semi-artinian absolutely flat rings and holds for certain semi-artinian absolutely flat rings, but have made no statements concerning general semi-artinian absolutely flat rings (there are certainly examples not covered by our current hypothesis). It turns out that the local-to-global principle holds for any semi-artinian absolutely flat ring, not just those we are currently considering, via a general argument making no use of an auxiliary noetherian ring. This result will appear, as a result of more general considerations, in future work.

From Lemma 4.20 we deduce a classification theorem for localising subcategories of $\mathrm{D}(R)$.

Theorem 4.23. Let $S$ be a ring with noetherian spectrum, and set $R=S^{\text {abs }}$. There is an order-preserving bijection

$$
\{\text { subsets of } \operatorname{Spec} R\} \underset{\sigma}{\stackrel{\tau}{\rightleftarrows}}\{\text { localising subcategories of } \mathrm{D}(R)\},
$$

where for a localising subcategory $\mathcal{L}$ and a subset $W \subseteq \operatorname{Spec} R$ we set

$$
\sigma(\mathcal{L})=\{P \in \operatorname{Spec} R \mid k(P) \otimes \mathcal{L} \neq 0\} \quad \text { and } \quad \tau(W)=\langle k(P) \mid P \in W\rangle .
$$

Proof. The map $\tau$ is a split monomorphism with left inverse $\sigma$ by [Ste13, Proposition 6.3]. Thus to prove the result we just need to observe that

$$
\begin{aligned}
\tau \sigma(\mathcal{L}) & =\tau(\{P \in \operatorname{Spec} R \mid k(P) \otimes \mathcal{L} \neq 0\}) \\
& =\langle k(P) \mid k(P) \otimes \mathcal{L} \neq 0\rangle,
\end{aligned}
$$

which is precisely $\mathcal{L}: \tau \sigma(\mathcal{L}) \subseteq \mathcal{L}$ is immediate as every localising subcategory of $\mathrm{D}(R)$ is a $\otimes$-ideal and $\mathcal{L} \subseteq \tau \sigma(\mathcal{L})$ is a straightforward consequence of Lemma 4.20. 
Thus Neeman's classification [Nee92] extends to the absolutely flat approximations of rings with noetherian spectrum. As a consequence, we see that, in contrast with the counterexample of Corollary 4.10, all the localising subcategories of such a ring are Bousfield classes.

Corollary 4.24. Let $R$ be as in the theorem. Every localising subcategory of $\mathrm{D}(R)$ is a Bousfield class. More precisely, given a subset $W \subseteq \operatorname{Spec} R$ with complement $V$ there is an equality

$$
\tau(W)=\operatorname{ker}\left(\bigoplus_{P \in V} k(P) \otimes-\right) .
$$

Proof. We have

$$
\begin{aligned}
\sigma\left(\operatorname{ker}\left(\bigoplus_{P \in V} k(P) \otimes-\right)\right) & =\left\{Q \in \operatorname{Spec} R \mid k(Q) \otimes \operatorname{ker}\left(\bigoplus_{P \in V} k(P) \otimes-\right) \neq 0\right\} \\
& =\operatorname{Spec} R \backslash V \\
& =W .
\end{aligned}
$$

Thus, invoking the theorem, we have $\tau(W)=\operatorname{ker}\left(\bigoplus_{P \in V} k(P) \otimes-\right)$, as claimed.

Given our classification result, it is natural to ask if we can settle the telescope conjecture in this setting. That it holds is an easy consequence of the formal results we have proved in Section 2.3.

Theorem 4.25. Let $S$ be a ring with noetherian spectrum, and set $R=S^{\text {abs }}$. The telescope conjecture holds for $\mathrm{D}(R)$.

Proof. Suppose $\mathcal{S}$ is a smashing subcategory of $\mathrm{D}(R)$, and let $\mathcal{S}^{\perp}$ be its right orthogonal, which is also a localising subcategory. By the theorem $\mathcal{S}$ and $\mathcal{S}^{\perp}$ are determined by the subsets $\sigma(\mathcal{S})$ and $\sigma\left(\mathcal{S}^{\perp}\right)$ of Spec $R$. One can easily check

$$
\sigma \mathcal{S}=\operatorname{Spec} R \backslash \sigma \mathcal{S}^{\perp}
$$

(cf. [Ste13, Lemma 7.13]). By Proposition 2.14 the subset $\sigma \mathcal{S}^{\perp}$ is proconstructible in $\operatorname{Spec} R$ and hence is closed as $\operatorname{Spec} R$ is already equipped with the constructible topology. Thus $\sigma \mathcal{S}$ is open and hence Thomason by Lemma 3.4. This shows $\mathcal{S}$ is compactly generated as, again using the theorem, we have

$$
\mathcal{S}=\tau(\sigma \mathcal{S})=\Gamma_{\sigma \mathcal{S}} \mathrm{D}(R)=\left\langle\mathrm{D}_{\sigma \mathcal{S}}^{\text {perf }}(R)\right\rangle .
$$

\subsection{Absolutely flat schemes}

We indicate here how to extend the results we have obtained in Section 4.2 to the analogous class of schemes. This involves no extra work, as the formalism of tensor actions allows us to deduce global results affine locally.

Given a scheme $X$, one can globalise Theorem 3.8 (see [Oli67b]) to obtain a universal map of schemes $X^{\text {abs }} \longrightarrow X$ where $X^{\text {abs }}$ is an absolutely flat scheme; i.e., $X^{\text {abs }}$ admits an open affine cover by the spectra of absolutely flat rings. For any open affine subscheme Spec $S$ of $X$, its preimage in $X^{\text {abs }}$ is just $\operatorname{Spec} S^{\text {abs }}$. 
Theorem 4.26. Let $X$ be a topologically noetherian scheme. The action of $\mathrm{D}(X)$ (or $\left.\mathrm{D}\left(X^{\mathrm{abs}}\right)\right)$ on $\mathrm{D}\left(X^{\mathrm{abs}}\right)$ gives an order-preserving bijection

$$
\left\{\text { subsets of } X^{\text {abs }}\right\} \underset{\sigma}{\stackrel{\tau}{\rightleftarrows}}\left\{\text { localising ideals of } \mathrm{D}\left(X^{\mathrm{abs}}\right)\right\} \text {, }
$$

where for a localising ideal $\mathcal{L}$ and a subset $W \subseteq X^{\text {abs }}$ we set

$$
\sigma(\mathcal{L})=\left\{x \in X^{\text {abs }} \mid k(x) \otimes \mathcal{L} \neq 0\right\} \quad \text { and } \quad \tau(W)=\langle k(x) \mid x \in W\rangle_{\otimes} .
$$

Proof. Given Theorem 4.23, one just chooses an open affine cover of $X^{\text {abs }}$ and applies [Ste13, Theorem 8.11].

Theorem 4.27. Let $X$ be a topologically noetherian scheme. The relative telescope conjecture holds for the action of $\mathrm{D}\left(X^{\text {abs }}\right)$ on itself; i.e., every smashing tensor ideal of $\mathrm{D}\left(X^{\mathrm{abs}}\right)$ is generated by objects of $\mathrm{D}^{\text {perf }}\left(X^{\mathrm{abs}}\right)$.

Proof. Again this follows from the result in the affine case, namely, Theorem 4.25. One chooses an open affine cover for $X^{\text {abs }}$ and applies [BF11, Theorem 6.6].

\section{References}

[Bal05] P. Balmer, The spectrum of prime ideals in tensor triangulated categories, J. Reine Angew. Math. 588 (2005), 149-168.

[BF11] P. Balmer and G. Favi, Generalized tensor idempotents and the telescope conjecture, Proc. Lond. Math. Soc. (3) 102 (2011), no. 6, 1161-1185.

[BIK11] D. Benson, S. B. Iyengar, and H. Krause, Stratifying triangulated categories, J. Topol. 4 (2011), no. 3, 641-666.

[BN93] M. Bökstedt and Amnon Neeman, Homotopy limits in triangulated categories, Compositio Math. 86 (1993), no. 2, 209-234.

[CS96] J. Clark and P.F. Smith, On semi-Artinian modules and injectivity conditions, Proc. Edinburgh Math. Soc. (2) 39 (1996), no. 2, 263-270.

[DP08] W.G. Dwyer and J.H. Palmieri, The Bousfield lattice for truncated polynomial algebras, Homology Homotopy Appl. 10 (2008), no. 1, 413-436.

[Fox79] H.-B. Foxby, Bounded complexes of flat modules, J. Pure Appl. Algebra 15 (1979), no. 2, $149-172$.

[Hoc69] M. Hochster, Prime ideal structure in commutative rings, Trans. Amer. Math. Soc. 142 (1969), 43-60.

[Hov95] M. Hovey, Cohomological Bousfield classes, J. Pure Appl. Algebra 103 (1995), no. 1, 45-59.

[HP99] M. Hovey and John H. Palmieri, The structure of the Bousfield lattice, Homotopy invariant algebraic structures (Baltimore, MD, 1998), 1999, pp. 175-196.

[Kra05] H. Krause, Cohomological quotients and smashing localizations, Amer. J. Math. 127 (2005), no. $6,1191-1246$.

[MV73] G.O. Michler and O.E. Villamayor, On rings whose simple modules are injective, J. Algebra 25 (1973), 185-201.

[Nee00] A. Neeman, Oddball Bousfield classes, Topology 39 (2000), no. 5, 931-935.

[Nee01] _ Triangulated categories, Annals of Mathematics Studies, vol. 148, Princeton University Press, Princeton, NJ, 2001.

[Nee92] — The chromatic tower for $D(R)$, Topology 31 (1992), no. 3, 519-532. With an appendix by Marcel Bökstedt.

[Nee96] _ The Grothendieck duality theorem via Bousfield's techniques and Brown representability, J. Amer. Math. Soc. 9 (1996), no. 1, 205-236.

[Oli67a] J.-P. Olivier, Anneaux absolument plats universels et épimorphismes à buts réduits, Séminaire Samuel. Algébre commutative 2 (1967), 1-12.

[Oli67b] L L foncteur $T^{-\infty}$. Globalisation du foncteur $T$, Séminaire Samuel. Algébre commutative 2 (1967), 1-10. 
[Sta14] The Stacks Project Authors, Stacks Project, 2014. http://stacks.math.columbia.edu.

[Ste13] G. Stevenson, Support theory via actions of tensor triangulated categories, J. Reine Angew. Math. 681 (2013), 219-254.

[Tho97] R.W. Thomason, The classification of triangulated subcategories, Compositio Math. 105 (1997), no. 1, 1-27.

[Tre06] M. Tressl, Computation of the z-radical in $C(X)$, Adv. Geom. 6 (2006), no. 1, 139-175.

[Tr196] J. Trlifaj, Two problems of Ziegler and uniform modules over regular rings, Abelian groups and modules (Colorado Springs, CO, 1995), 1996, pp. 373-383.

\section{Greg Stevenson gstevens@math.uni-bielefeld.de}

Universität Bielefeld, Fakultät für Mathematik, BIREP Gruppe, Postfach 100131 , 33501 Bielefeld, Germany 